\title{
Viewpoint
}

\section{Materials Prediction Scores a Hit}

\author{
Filip Ronning and John L. Sarrao \\ Los Alamos National Laboratory, Los Alamos, New Mexico 87545, USA \\ Published October 7, 2013
}

Calculations predicting a new high-pressure superconductor are borne out by experiment.

\author{
Subject Areas: Materials Science
}

\author{
A Viewpoint on: \\ Discovery of a Superhard Iron Tetraboride Superconductor \\ Huiyang Gou, Natalia Dubrovinskaia, Elena Bykova, Alexander A. Tsirlin, Deepa Kasinathan, Walter Schnelle, Asta \\ Richter, Marco Merlini, Michael Hanfland, Artem M. Abakumov, Dmitry Batuk, Gustaaf Van Tendeloo, Yoichi \\ Nakajima, Aleksey N. Kolmogorov, and Leonid Dubrovinsky \\ Phys. Rev. Lett. 111, 2013 - Published October 7, 2013
}

Had the great American philosopher Yogi Berra been a condensed matter physicist, he might have said "It's difficult to make predictions, especially about superconductivity." Predictions about a material's structure and even more so its function have been goals of materials research for a long time, but the track record for predicting that a given compound will superconduct is notoriously bad [1]. Fortunately, advances in the fidelity and resolution of electronic structure calculations are beginning to change this trend [2]. In fact, the White House's Materials Genome Initiative [3] represents a recognition that with recent advances in computational capability and materials models, such breakthroughs are possible and, in fact, likely probable. In a paper in Physical Review Letters, Huiyang Gou at the University of Bayreuth, Germany, and colleagues [4] describe a success story in the search for predictability. They report the observation of superconductivity in iron tetraboride $\left(\mathrm{FeB}_{4}\right)$ at approximately 3 kelvin (K). Not only did they find superconductivity where electronic structure calculations told them to look, they used high-pressure synthesis techniques to discover a compound that wasn't readily apparent in nature. Further, the resulting compound, orthorhombic $\mathrm{FeB}_{4}$, turns out to be very mechanically hard as well as superconducting, thus possessing two desirable traits.

Most attempts to predict superconductivity invoke the physicist Bernd Matthias [5]. In the 1950s-1970s Matthias articulated a number of empirical "rules" that anticipated a large number of superconducting materials based on crystal structure and the number of valence electrons per atom. However, these rules were clearly based on intuition and not predictive theory. The experimental discovery that cuprates, magnesium diboride $\left(\mathrm{MgB}_{2}\right)$, and more recently, iron pnictides all superconduct drove home the reality that serendipity was still the best materials discovery engine. However, that reality is

DOI: 10.1103/Physics.6.109

URL: http://link .aps .org/doi/10.1103/Physics.6.109 beginning to evolve.

Why is it so difficult to predict new superconducting materials? One issue is the difficulty predicting the structural stability of a compound, that is, whether the binding energy between atoms is large enough to keep them stuck together in a particular configuration. Electronic structure calculations provide the total energy for a crystal, which is on the order of $10^{5}$ electron volts per atom (eV/atom) (see Fig. 1). However, the stability with respect to competing phases is typically as small as $10^{-2}$ $\mathrm{eV} /$ atom, thus demanding incredibly high accuracy of the calculations. Furthermore, calculations are typically performed at $T=0 \mathrm{~K}$ in ideal crystals, while the thermal energy at which the crystals are synthesized and the energy scale created by defects can easily shift the relative total energies of competing phases by similar amounts. Another factor is that superconductivity is a very lowenergy instability of the electronic structure. For a superconductor with a transition temperature $T_{c}$ of $3 \mathrm{~K}$, as discovered by Gou et al., this amounts to an energy scale of $10^{-4} \mathrm{eV}$. Few predictive models (yet) have accuracy at the parts per billion level covered by these energy scales.

Advanced electronic structure calculations for predictions have increased effectiveness due to the relative accessibility and availability of high-pressure techniques. Recent discoveries demonstrate that surprises still exist at high pressure [6]. We now know that a dozen or so additional elements superconduct at elevated pressure even though they are normal materials under ambient conditions, including calcium at 220 gigapascals (with $T_{c}=29 \mathrm{~K}$, the highest $T_{c}$ for an elemental superconductor). More broadly, materials science has been transformed by our ability to apply sufficient pressure to tune structural energetics on this scale to make new states of matter available.

In 2010, Kolmogorov, a coauthor of the present 


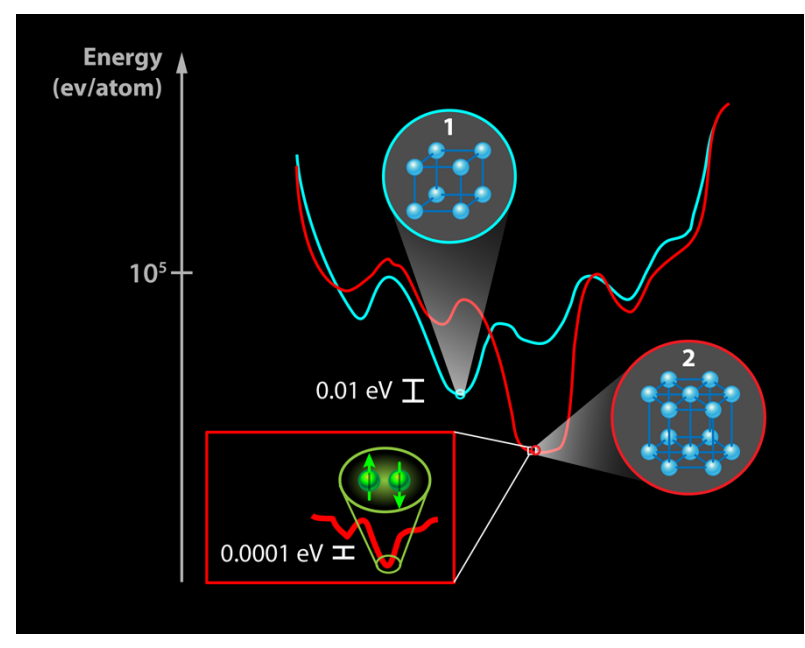

FIG. 1: The energetics of predicting materials. A schematic free energy landscape for different crystallographic configurations is given by the blue line. Note the small difference in energy between various structures compared with the total energy of a crystal demanding high computational accuracy. The application of pressure as done by Gou et al. will modify the energy landscape (red curve), potentially stabilizing new structures. The ground state (such as superconductivity, magnetism, or other forms of order) for a given structure is determined at even lower energy scales, as depicted in the inset. The addition of strong electronic correlations in some materials will further modify the landscape over large energy scales up to $10 \mathrm{eV}$, making predictions even more challenging. (APS/Alan Stonebraker)

study, and colleagues predicted additional phases in the iron-boron $(\mathrm{Fe}-\mathrm{B})$ binary phase diagram that had yet to be observed [7]. They used a high-throughput search method coupled to an evolutionary algorithm to identify new structures for which superconductivity was theoretically evaluated. Subsequently, Bialon et al. suggested that the stability of iron tetraboride $\left(\mathrm{FeB}_{4}\right)$ would be enhanced under pressure, and predicted the material's hardness [8]. In the present manuscript, Gou et al. confirmed that $\mathrm{FeB}_{4}$ can be synthesized under pressure, and furthermore, that it possess the two novel predicted properties: superconductivity and high incompressibility. In addition, though not definitive, Gou et al. obtained preliminary data that superconductivity is phonon mediated like other conventional superconductors.

While the paper by Gou et al. gives promise that theory may finally be able to guide experimentalists where to look for conventional superconductors, it's important to remember that the predicted $T_{c}$ was 5 times too large in a structure that couldn't be synthesized at ambient pressure. Further, the situation remains much more challenging for unconventional superconductors such as the cuprates, pnictides, heavy fermion materials, and organics. The biggest issue is that strong electronic correlations alter the electronic structure in these materials over an energy scale of order 1-10 eV. While modern electronic structure calculations such as dynamical mean- field theory are making progress in understanding these effects, we currently lack the ability to reliably identify an additional superconducting instability on this strongly correlated background. How these electronic correlations modify the ability to compute structural stability of compounds also remains an open question. Given that superconductivity emerges in strongly correlated systems in ways we least expect it 9], future searches would be aided by guidance on where to find such correlations and competing electronic instabilities.

Gou et al. provide an encouraging step in the quest for materials by design, but one can also hope that this is a harbinger of even more and better things to come. Leveraging advanced computational capabilities and associated materials algorithms, together with synthetic techniques that allow broader access to phase space, including metastable materials, holds the exciting potential of delivering on the vision of the Materials Genome Initiative. We look forward to this, bearing in mind the quote attributed to Yogi Berra: "It's difficult to make predictions, especially about the future."

\section{Acknowledgment}

Our work in this area has been supported by the Department of Energy's Office of Basic Energy Sciences Division of Materials Science and Engineering.

\section{References}

[1] I. I. Mazin, "Superconductivity Gets an Iron Boost," Nature 464, 183 (2010).

[2] R. Akashi and R. Arita, "Development of Density-Functional Theory for a Plasmon-Assisted Superconducting State: Application to Lithium Under High Pressures," Phys. Rev. Lett. 111, 057006 (2013).

[3] Materials Genome Initiative for Global Competitiveness, www.whitehouse.gov/blog/2011/06/24/materials-genomeinitiative-renaissance-american-manufacturing.

[4] H. Gou et al., "Discovery of a Superhard Iron Tetraboride Superconductor," Phys. Rev. Lett. 111, 157002 (2013).

[5] B. T. Matthias, T. H. Geballe, and V. B. Compton, "Superconductivity," Rev. Mod. Phys. 35, 1 (1963).

[6] M. Sakata, Y. Nakamoto, K. Shimizu, T. Matsuoka, and Y. Ohishi, "Superconducting state of Ca-VII below a critical temperature of $29 \mathrm{~K}$ at a pressure of $216 \mathrm{GPa}$," Phys. Rev. B 83, 220512(R) (2011).

[7] A. N. Kolmogorov, S. Shah, E. R. Margine, A. F. Bialon, T. Hammerschmidt, and R. Drautz, "New Superconducting and Semiconducting Fe-B Compounds Predicted with an Ab Initio Evolutionary Search," Phys. Rev. Lett. 105, 217003 (2010).

[8] A. F. Bialon, T. Hammerschmidt, R. Drautz, S. Shah, E. R. Margine, and A. N. Kolmogorov, "Possible Routes for Synthesis of New Boron-Rich Fe-B and $\mathrm{Fe}_{1-x} \mathrm{Cr}_{x} \mathrm{~B}_{4}$ Compounds," Appl. Phys. Lett. 98, 081901 (2011).

[9] Z. Fisk, H. R. Ott, and J. D. Thompson, "Superconducting materials: What the record tells us," Philos. Mag. 89, 2111 (2009). 


\section{About the Authors}

\section{Filip Ronning}

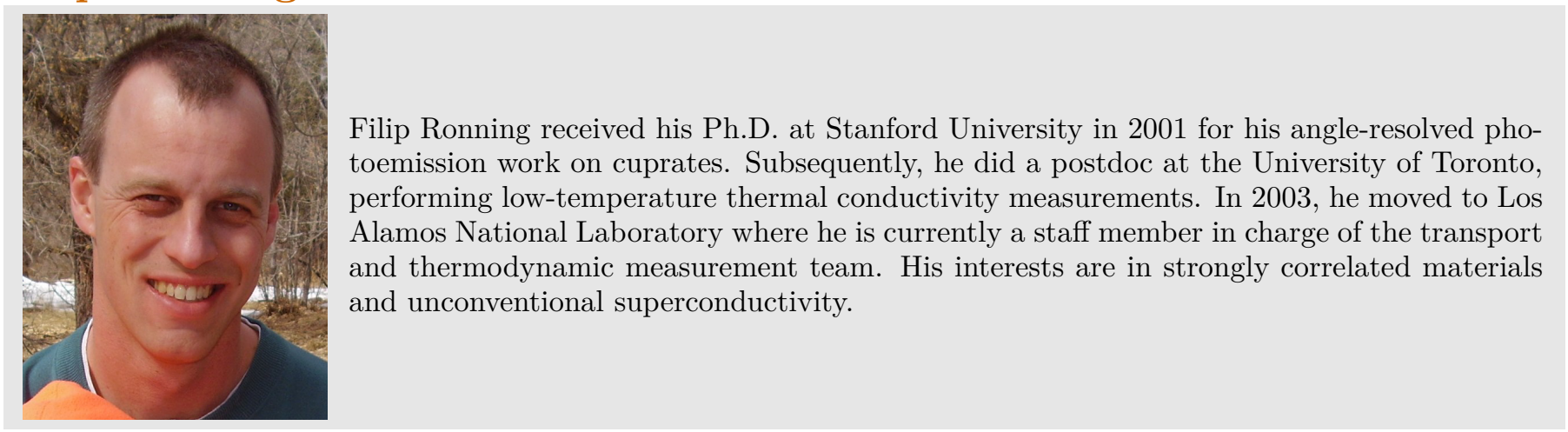

\section{John L. Sarrao}

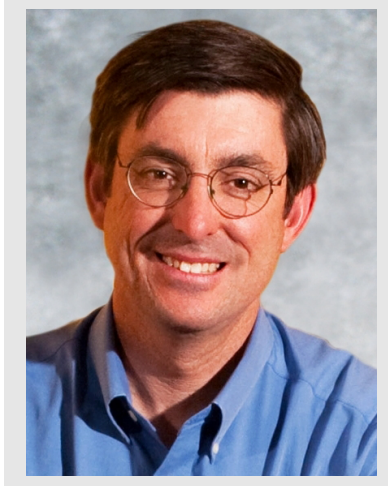

John Sarrao is the Associate Director for Theory, Simulation, and Computation at Los Alamos National Laboratory. His own research is focused on the discovery, synthesis, and characterization of correlated electron systems, especially actinide materials and novel superconductors. He received his Ph.D. from the University of California, Los Angeles, in 1993, based on work done at Los Alamos. He is a Fellow of the American Physical Society (APS), the American Association for the Advancement of Science (AAAS), and Los Alamos National Laboratory. 